\title{
Newton Law on the Generalized Singular Brane with and without 4d Induced Gravity
}

\author{
Eylee Jung*, SungHoon Kim ${ }^{\dagger}$, and D. K. Park ${ }^{\ddagger}$, \\ Department of Physics, Kyungnam University, Masan, 631-701, Korea
}

\begin{abstract}
Newton law arising due to the gravity localized on the general singular brane embedded in $A d S_{5}$ bulk is examined in the absence or presence of the $4 d$ induced Einstein term. For the RS brane, apart from the subleading correction, Newton potential obeys $4 d$-type and $5 d$-type gravitational law at long- and short-ranges if it were not for the induced Einstein term. The $4 d$ induced Einstein term generates an intermediate range at short distance, in which the $5 d$ Newton potential $1 / r^{2}$ emerges. For Neumann brane the long-range behavior of Newton potential is exponentially suppressed regardless of the existence of the induced Einstein term. For Dirichlet brane the expression of Newton potential is dependent on the renormalized coupling constant $v^{r e n}$. At particular value of $v^{\text {ren }}$ Newton potential on Dirichlet brane exhibits a similar behavior to that on RS brane. For other values the long-range behavior of Newton potential is exponentially suppressed as that in Neumann brane.
\end{abstract}

\footnotetext{
*Email:eylee@mail.kyungnam.ac.kr

†Email:shoon@mail.kyungnam.ac.kr

‡Email:dkpark@hep.kyungnam.ac.kr
} 


\section{INTRODUCTION}

A braneworld scenario is a physical picture which assumes that our $4 d$ spacetime universe is embedded in higher-dimensional world. Although higher-dimensional theory has its own long history [1-3] in the context of Kaluza-Klein theory or not, modern braneworld scenarios such as large extra dimensions [4,5] or warped extra dimensions $[6,7]$ seem to be mainly motivated from the string theories [8]. Making use of the recent scenarios a flurry of activities has tried to examine the various physical problems such as big bang universe [9-11], cosmological constant hierarchy [12-14], brane inflation [15], and black hole physics [16-21]. In this paper we will examine Newton law arising due to the gravity localized on the brane when general singular brane is embedded in $A d S_{5}$ bulk.

The Newton law on the brane was firstly computed by RS in Ref. [7] when the bulk spacetime is $A d S_{5}$. In this case the gravity localized on the brane yields an $4 d$-type $1 / r$ and $1 / r^{3}$ subleading correction arising due to Kaluza-Klein exictation:

$$
V \sim G \frac{m_{1} m_{2}}{r}\left(1+\frac{R^{2}}{r^{2}}\right)
$$

where $R$ is a radius of $A d S_{5}$. Under the same setup an improvement of the potential was tried by involving the brane-bending effect $[18,22]$ and by computing the one-loop correction to the gravitational propagator [23], which slightly changes the sub-leading correction by a overall multiplication factor

$$
V \sim G \frac{m_{1} m_{2}}{r}\left(1+\frac{2}{3} \frac{R^{2}}{r^{2}}\right)
$$

Subsequently, the gravitational propagator for the linearized fluctuation equation in RS picture is generally computed by adopting the singular quantum mechanics(SQM) as a calculational tool $[24,25]$. From the viewpoint of SQM the linearized gravitational propagator is crucially dependent on the boundary condition(BC) that the propagator should satisfy at the location of the brane. The physically relevant $\mathrm{BC}$ is not uniquely determined in the framework of SQM due to the singular nature of the potential and the physically consistent 
BCs are parametrized by a real parameter, say $\xi$. The physically consistent BCs are in general introduced by a self-adjoint extension technique [26,27], which is effectively identical to the coupling constant renormalization [28,29]. Recently, this generalized gravitational propagators are used to compute the Newton potential on the brane [30,31] with a particular choice of $\xi$ as $\xi=1 / 2$, which means the Dirichlet and Neumann BCs are included with an equal weight.

Recently, the Newton law with different setup is also considered. Especially, much attention is paid to the case of Minkowski bulk with an involving the $4 d$ induced Einstein term arising due to the quantum effect of one loop $[32,33]$. In the case of the flat bulk the Newton potential becomes $4 d$-type $1 / r$ at the short range, i.e. $r<<\lambda$, and $5 d$-type $1 / r^{2}$ at the long range, i.e. $r>>\lambda$, where $\lambda$ is a ratio of $4 d$ Planck scale with that of $5 d ; \lambda \equiv M_{4}^{2} / M_{5}^{3}$. This fact is used to explain the observed acceleration of the Universe [34].

The effect of the induced Einstein term is also examined when the $5 d$ bulk is $A d S_{5}$ spacetime $[35,36]$. In this setup the $5 d$-type Newton potential arises in the region of $\lambda<<$ $r<<R$. At other ranges the $4 d$ gravitational potential is recovered. Recently, this fact is again confirmed in the context of SQM [31].

In this paper we will examine the Newton potential using the generalized gravitational propagators derived using SQM. Thus, the gravittational propagator contains in general the parameter $\xi$, which parametrizes the physically relevant BCs. The $\xi$-dependence of the Newton potential is examined throughout the paper. We will confine ourselves to the case of single copy of the $A d S_{5}$ bulk. Thus our computation is limited to the original RS plus $A d S / C F T[37,38]$ setup with and without the induced Einstein term. In particular, we will examine in detail the cases of $\xi=0, \xi=1$, and $\xi=1 / 2$. When $\xi=1$, we have a Neumann brane in which the gravity acquires mass [24,25]. It is well-known that the Newton potential generated due to the exchange of a massive graviton is exponentially suppressed at long range. This fact will be explicitly shown in the following. At $\xi=0$ we have a Dirichlet brane. In this case the non-trivial gravitational propagator can be derived via the coupling constant renormalization [24,25]. Thus the final expression of the Newton 
potential is dependent on the renormalized coupling constant. We will show in the following that when the renormalized coupling constant has a particular valus, the Newton potential on the Dirichlet brane is proportional to that on RS brane which corresponds to the brane at $\xi=1 / 2$.

The paper is origanized as following. In Sec.II we will derive a formula which shows how to compute Newton potential arising due to a localized gravity from the fixed-energy amplitude. Using the formula Newton law in general singular brane is examined in Sec.III without consideration of the $4 d$ induced Einstein term when bulk is a single copy of $A d S_{5}$ spacetime. A particular attention is paid to the $\operatorname{RS}-b r a n e(\xi=1 / 2)$, Neumann brane $(\xi=1)$ and Dirichlet brane $(\xi=0)$. The effect of the $4 d$ induced Einstein term to Newton potential is studied in Sec.IV. In Sec.V brief conclusion and further remark are given. In appendix A and B Newton laws on Neumann and Dirichlet branes are explicitly derived in the presence of the $4 d$ induced Einstein term.

\section{NEWTON POTENTIAL FROM THE FIXED-ENERGY AMPLITUDE}

In this section we will consider briefly how to compute Newton potential arising due to the localized gravity on the brane from the fixed-energy amplitude ${ }^{1}$ computed in the context of the SQM. As an example we will consider the usual RS scenario without the induced Einstein term. However, the final result is model-independent.

Let us consider the $5 d$ Einstein equation ${ }^{2}$

$$
R_{M N}-\frac{1}{2} G_{M N} R=-\frac{1}{4 M^{3}}\left[\Lambda G_{M N}+v_{b} G_{\mu \nu} \delta_{M}^{\mu} \delta_{N}^{\nu} \delta(y)\right]
$$

where $M, \Lambda$ and $v_{b}$ are $5 d$ Planck scale, $5 d$ cosmological constant and brane tension respec-

\footnotetext{
${ }^{1}$ The definition of the fixed-energy amplitude is a Laplace transform of the Euclidean propagator.

${ }^{2}$ Our notation is that $M$ and $N$ are bulk spacetime indices and, $\mu$ and $\nu$ are brane spacetime indices.
} 
tively. As is well-known it has a solution

$$
d s^{2}=e^{-2 k|y|} \eta_{\mu \nu} d x^{\mu} d x^{\nu}+d y^{2}
$$

if the fine-tuning conditions $\Lambda=-24 M^{3} k^{2}$ and $v_{b}=24 M^{3} k$ are satisfied.

Introducing the linearized gravitational fluctuation $h_{\mu \nu}(x, y)$ as following

$$
d s^{2}=\left[e^{-2 k|y|} \eta_{\mu \nu}+h_{\mu \nu}(x, y)\right] d x^{\mu} d x^{\nu}+d y^{2}
$$

and ignoring the tensor structure for simplicity by choosing a gauge $h_{\mu \nu, \mu}=h_{\mu}^{\mu}=0$, one can derive the following linearized fluctuation equation

$$
\left[e^{2 k|y|} \square^{(4)}+\partial_{y}^{2}-4 k^{2}+4 k \delta(y)\right] h_{\mu \nu}=0
$$

which reduces to

$$
\begin{aligned}
& \hat{H}_{R S} \hat{\psi}(z)=\frac{m^{2}}{2} \hat{\psi}(z) \\
& \hat{H}_{R S}=-\frac{1}{2} \partial_{z}^{2}+\frac{15}{8(|z|+R)^{2}}-\frac{3}{2} k \delta(z)
\end{aligned}
$$

where $R \equiv 1 / k$ is a radius of $A d S_{5}$ and

$$
\begin{aligned}
& z=\epsilon(y) \frac{e^{k|y|}-1}{k} \\
& h_{\mu \nu}(x, y)=e^{-\frac{k}{2}|y|} \hat{\psi}(y) e^{i p x} \\
& m^{2}=-p^{2} .
\end{aligned}
$$

At this stage we should stress the fact that if our calculation involves the brane-bending effect, Eq.(2.4) is modified into

$$
\left[e^{2 k|y|} \square^{(4)}+\partial_{y}^{2}-4 k^{2}+4 k \delta(y)\right] h_{\mu \nu}=-\Sigma_{\mu \nu} \delta(y)
$$

where $\Sigma_{\mu \nu}$ is a tensor quantity and represents the brane-bending effect [22]. Thus, the additional term plays the role of the source and recovers the tensor structure of $h_{\mu \nu}$. Although it might be possible, in principle, to compute the propagator of Eq.(2.7) from the viewpoint of SQM with imposing a general $\mathrm{BC}$ on the brane after computing $\Sigma_{\mu \nu}$ explicitly and changing 
Eq.(2.7) as a Schrödinger-type equation, it may lead an extreme complication. Since our interest is to examine the effect of the general boundary condition in the Newton potential, we will confine ourselves to Eq.(2.5) without recovering the tensor structure.

The Newton potential in the bulk is in general computed by the time-integration of the retarded Green function $U\left(\vec{x}_{2}, y_{2} ; \vec{x}_{1}, y_{1} ; t\right)[22,32]$ :

$$
V\left(\vec{x}_{2}, y_{2} ; \vec{x}_{1}, y_{1}\right) \equiv \int d t U\left(\vec{x}_{2}, y_{2} ; \vec{x}_{1}, y_{1} ; t\right)
$$

In terms of the time-dependent propagator, $U\left(\vec{x}_{2}, y_{2} ; \vec{x}_{1}, y_{1} ; t\right)$ is represented as

$$
U\left(\vec{x}_{2}, y_{2} ; \vec{x}_{1}, y_{1} ; t\right)=\frac{1}{M^{3}} \int \frac{d^{4} p}{(2 \pi)^{3}} e^{-\frac{k}{2}\left(\left|y_{1}\right|+\left|y_{2}\right|\right)} G\left[y_{1}+R, y_{2}+R ; t\right] e^{i \vec{p} \cdot\left(\vec{x}_{2}-\vec{x}_{1}\right)} e^{-p_{0} t} \delta\left(p_{0}-|\vec{p}|\right) .
$$

The factor $e^{-\frac{k}{2}\left(\left|y_{1}\right|+\left|y_{2}\right|\right)}$ in Eq.(2.9) is introduced due to the redefinition of $h_{\mu \nu}(x, y)$ in Eq.(2.6). The time-integration in Eq.(2.8) changes the Euclidean propagator $G\left[y_{1}+R, y_{2}+\right.$ $R ; t]$ into the fixed-energy amplitude $\hat{G}\left[y_{1}+R, y_{2}+R ; \frac{p_{0}^{2}}{2}\right]$ as following:

$$
V\left(\vec{x}_{2}, y_{2} ; \vec{x}_{1}, y_{1}\right)=\frac{1}{8 \pi^{3} M^{3}} e^{-\frac{k}{2}\left(\left|y_{1}\right|+\left|y_{2}\right|\right)} \int d^{4} p e^{i \vec{p} \cdot\left(\vec{x}_{2}-\vec{x}_{1}\right)} \delta\left(p_{0}-|\vec{p}|\right) \hat{G}\left[y_{1}+R, y_{2}+R ; \frac{p_{0}^{2}}{2}\right]
$$

Thus the $p_{0}$-integration in Eq.(2.10) results in

$$
V\left(\vec{x}_{2}, y_{2} ; \vec{x}_{1}, y_{1}\right)=\frac{1}{8 \pi^{3} M^{3}} e^{-\frac{k}{2}\left(\left|y_{1}\right|+\left|y_{2}\right|\right)} \int d^{3} \vec{p} e^{i \vec{p} \cdot\left(\vec{x}_{2}-\vec{x}_{1}\right)} \hat{G}\left[y_{1}+R, y_{2}+R ; \frac{\vec{p}^{2}}{2}\right] .
$$

If the brane has three spatial dimensions and is located at $y=0$, the Newton potential on the brane is expressed as

$$
V(r) \equiv V\left(\left|\vec{x}_{2}-\vec{x}_{1}\right|, y_{1}=y_{2}=0\right)=\frac{1}{2 \pi^{2} M^{3} r} \int_{0}^{\infty} d m m \sin m r \hat{G}\left[R, R ; \frac{m^{2}}{2}\right]
$$

where $r \equiv\left|\vec{x}_{2}-\vec{x}_{1}\right|$.

Eq.(2.12) enables us to compute the Newton potential generated by the localized gravity on the brane from the fixed-energy amplitude $\hat{G}\left[R, R ; \frac{m^{2}}{2}\right]$. Since the general fixed-energy amplitude which depends on the BC is computed Ref. [24,25] when there is no $4 d$ induced Einstein term, we can compute the Newton potential by making use of Eq.(2.12). This will be examined in the next section in detail. 


\section{NEWTON POTENTIAL FROM FIXED-ENERGY AMPLITUDE : WITHOUT $4 D$ INDUCED EINSTEIN TERM}

In this section we will compute the Newton potential arising due to the localized gravity on the brane when there is no $4 d$ induced Einstein term. Thus what we have to do first is to derive the fixed-energy amplitude for the linearized gravitational fluctuation (2.5). In Ref. [24,25] Eq.(2.5) is slightly generalized as following

$$
\begin{aligned}
& \hat{H}_{1} \hat{\psi}(z)=E \hat{\psi}(z) \\
& \hat{H}_{1}=\hat{H}_{0}-v \delta(z) \\
& \hat{H}_{0}=-\frac{1}{2} \partial_{z}^{2}+\frac{g}{(|z|+c)^{2}} .
\end{aligned}
$$

Of course, $\hat{H}_{1}$ in Eq.(3.1) coincides with $\hat{H}_{R S}$ in Eq.(2.5) when $c=1 / k \equiv R, g=15 / 8$, and $v=3 k / 2$.

As we commented before we require the bulk spacetime is a single copy of $A d S_{5}$. This requirement leads us naturally to combine the usual Schulman procedure $[39,40]$ for the treatment of the $\delta$-function potential with an half-line constraint. The half-line constraint usually makes the fixed-energy amplitude to be dependent on the $\mathrm{BC}$ on the brane. This procedure is explicitly addressed in Ref. [24,25]. The final expression of the fixed-energy amplitude for $\hat{H}_{1}$ under these circumstance is

$$
\begin{aligned}
\hat{G}_{1}[a, b ; E] & =\hat{G}_{0}^{D}[a, b ; E] \\
& +\frac{\sqrt{a b}}{c v} \frac{K_{\gamma}(\sqrt{2 E} a) K_{\gamma}(\sqrt{2 E} b)}{K_{\gamma}^{2}(\sqrt{2 E} c)}\left[\left(\frac{\gamma-\frac{1}{2}}{2 \xi c v}-1\right)+\frac{\sqrt{2 E}}{2 \xi v} \frac{K_{\gamma-1}(\sqrt{2 E} c)}{K_{\gamma}(\sqrt{2 E} c)}\right]^{-1}
\end{aligned}
$$

where $\gamma \equiv \sqrt{1+8 g} / 2$ and $K_{\gamma}(z)$ is an usual modified Bessel function. The parameter $\xi$ is explicitly introduecd in Eq.(3.2). The real parameter $\xi$ parametrizes the BCs for the fixed-energy amplitude corresponding to $\hat{H}_{0}$. For example, $\xi=1$ (or $\xi=0$ ) means that we choosed the Neumann (or Dirichlet) BC for the fixed-energy amplitude corresponding to $\hat{H}_{0} . \hat{G}_{0}^{D}[a, b ; E]$ in Eq.(3.2) is a fixed-energy amplitude for $\hat{H}_{0}$ when we adopt a Dirichlet BC at the brane. The explicit form of $\hat{G}_{0}^{D}[a, b ; E]$ is given in Ref. [24,25]. However we do 
not need to know its explicit form. The only one we should know is the fact that it satisfies the usual Dirichlet BC, i.e. $\hat{G}_{0}^{D}[a, c ; E]=\hat{G}_{0}^{D}[c, b ; E]=0$.

The fixed-energy amplitude on the brane $\hat{G}_{1}[c, c ; E]$ is easily computed from Eq.(3.2):

$$
\hat{G}_{1}[c, c ; E]=\frac{1}{v}\left[\left(\frac{\gamma-\frac{1}{2}}{2 \xi c v}-1\right)+\frac{\sqrt{2 E}}{2 \xi v} \frac{K_{\gamma-1}(\sqrt{2 E} c)}{K_{\gamma}(\sqrt{2 E} c)}\right]^{-1} .
$$

Thus inserting Eq.(3.3) into Eq.(2.12) we can explicitly compute the Newton potential.

Firstly, we consider $\xi=1 / 2$ case which corresponds to the case of usual RS brane. Letting $c=R, g=15 / 8$ and $v=3 / 2 R$ makes the fixed-energy amplitude to be

$$
\hat{G}_{1}^{R S}[R, R ; E]=\Delta_{0}+\Delta_{K K}
$$

where

$$
\begin{aligned}
\Delta_{0} & =\frac{1}{E R} \\
\Delta_{K K} & =\frac{1}{\sqrt{2 E}} \frac{K_{0}(\sqrt{2 E} R)}{K_{1}(\sqrt{2 E} R)} .
\end{aligned}
$$

Of course, $\Delta_{0}$ and $\Delta_{K K}$ represent the zero mode and KK excitation respectively. Inserting Eq.(3.4) into Eq.(2.12) it is easy to show that the Newton potential on the RS brane reduces to

$$
V_{R S}(r)=V_{0, R S}(r)+\Delta V_{R S}(r)
$$

where

$$
\begin{aligned}
V_{0, R S}(r) & =\frac{G}{r} \\
\Delta V_{R S}(r) & =\frac{G}{\pi} \frac{1}{r} \int_{0}^{\infty} d u \sin \left(\frac{r}{R} u\right) \frac{K_{0}(u)}{K_{1}(u)}
\end{aligned}
$$

and $4 d$ Newton constant is defined as $G \equiv 1 / 2 \pi M^{3} R$.

It is not difficult to show that the integral in $\Delta V^{R S}(r)$ is not well-defined. Thus we need an appropriate regularization. This is achieved by introducing the infinitesimal parameter $\epsilon$ as following $[31,35]$ 


$$
\Delta V_{R S}(r)=\frac{G}{\pi} \frac{1}{r} J
$$

where

$$
J \equiv \lim _{\epsilon \rightarrow 0} \int_{0}^{\infty} d u \sin \left(\frac{r}{R} u\right) \frac{K_{0}(u)}{K_{1}(u)} e^{-\epsilon u}
$$

The factor $\sin \left(\frac{r}{R} u\right)$ in Eq.(3.8) is crucial to extract the long-range behavior and shortrange behavior of the Newton potential. Firstly, let us consider the long-range behavior, i.e. $r>>R$. The high oscillation behavior of $\sin \left(\frac{r}{R} u\right)$ in this case makes the small $u$ region to be a dominant contribution. Since $K_{0}(u) \sim-\ln u$ and $K_{1}(u) \sim 1 / u$ at $u \sim 0, J$ becomes approximately in this region

$$
J \sim-\lim _{\epsilon \rightarrow 0} \int_{0}^{\infty} d u \sin \left(\frac{r}{R} u\right) u \ln u e^{-\epsilon u}
$$

Using an integration formula

$$
\begin{gathered}
\int_{0}^{\infty} d x x e^{-q x} \sin (p x) \ln x=\frac{1}{p^{2}+q^{2}} \sin \left(2 \tan ^{-1} \frac{p}{q}\right)\left[1-\gamma-\frac{1}{2} \ln \left(p^{2}+q^{2}\right)\right. \\
\left.+\tan ^{-1} \frac{p}{q} \cot \left(2 \tan ^{-1} \frac{p}{q}\right)\right]
\end{gathered}
$$

where $\gamma$ in Eq.(3.11) is an Euler's constant, it is simple to show $J \sim \pi R^{2} / 2 r^{2}$.

Secondly, let us consider the short-range behavior, i.e. $r<<R$. In this case contrary to the long-range behavior the large $u$ region makes a dominant contribution to $J$. Since at this region $K_{0}(u) \sim K_{1}(u) \sim \sqrt{\frac{\pi}{2 u}} e^{-u}, J$ reduces to

$$
J \sim \int_{0}^{\infty} d u \sin \left(\frac{r}{R} u\right) e^{-\epsilon u}
$$

which makes $J$ to be $R / r$. Thus we can summarize the behavior of $J$ as following:

$$
J \equiv \lim _{\epsilon \rightarrow 0} \int_{0}^{\infty} d u \sin \left(\frac{r}{R} u\right) \frac{K_{0}(u)}{K_{1}(u)} e^{-\epsilon u} \sim \begin{cases}\frac{\pi R^{2}}{2 r^{2}} & \text { if } r>>R \\ \frac{R}{r} & \text { if } r<<R .\end{cases}
$$

Eq.(3.13) is confirmed numerically. Fig. 1 shows that $J$ and $\pi R^{2} / 2 r^{2}$ at long-range. For simple numerical calculation we choosed $\epsilon=0.003$. Fig. 1 indicates that $J$ and $\pi R^{2} / 2 r^{2}$ 
merge with each other at $r \sim 15 R$. Of course, if we choosed smaller $\epsilon$, the mergence may occur rapidly. Fig. 2 shows that $J$ and $R / r$ at short-range with choosing $\epsilon=0.003$. Fig. 2 also indicates that $J$ and $R / r$ merge with each other at $r \sim 0.05 R$.

Using Eq.(3.13) one can show that the Newton potential on the RS brane becomes

$$
V_{R S}(r) \sim \begin{cases}\frac{G}{r}\left(1+\frac{R^{2}}{2 r^{2}}\right) & \text { if } r>>R \\ \frac{G R}{\pi r^{2}}\left(1+\frac{\pi r}{R}\right) & \text { if } r<<R .\end{cases}
$$

Thus we have $5 d$ Newton potential $1 / r^{2}$ at the short-range. Of course, the Newton potential is $4 d$-type $1 / r$ at long-range as shown in Ref. [7].

Here, we should point out that the long-range behavior of the subleading term of $V_{R S}(r)$ is different from both Eq.(1.1) and Eq.(1.2) due to 1/2 factor. In fact, the subleading term for our approach is $J / \pi$ and we computed $J$ with adopting the simple regularization scheme in Eq.(3.9). However, the different scheme may assign different value to $J$. In this way, the coefficient of the subleading term is dependent on the regularization scheme. It seems to be interesting to find a suitable regularization scheme which yields Eq.(1.1) or Eq.(1.2). Since, however, our interest in this paper is only to find a global behavior of Newton potential in general singular brane, we will not explore this issue further.

Now, we consider $\xi=1$ case which means that the Neumann BC is chosen. Inserting $c=R, g=15 / 8$ and $v=3 / 2 R$ into Eq.(3.3) one can show that the fixed-energy amplitude on the Neumann brane is

$$
\hat{G}_{1}^{N}\left[R, R ; \frac{m^{2}}{2}\right]=\frac{\frac{2}{m} \frac{K_{2}(m R)}{K_{1}(m R)}}{1-\frac{3}{2} \frac{1}{m R} \frac{K_{2}(m R)}{K_{1}(m R)}} .
$$

Inserting Eq.(3.15) into (2.12) shows that the Newton potential arising due to the localized gravity on the Neumann brane reduces to

$$
V_{N}(r)=\frac{1}{\pi^{2} M^{3} R r} \int_{0}^{\infty} d u \sin \left(\frac{r}{R} u\right) \frac{\frac{K_{2}(u)}{K_{1}(u)}}{1-\frac{3}{2 u} \frac{K_{2}(u)}{K_{1}(u)}} .
$$

Since the term $\sin \left(\frac{r}{R} u\right)$ makes that the small $u$ region contributes dominantly at the longrange, the Newton potential at this range becomes 


$$
V_{N}(r) \sim \frac{-2}{\pi^{2} M^{3} R r} \int_{0}^{\infty} d u \sin \left(\frac{r}{R} u\right) \frac{u}{3-u^{2}}
$$

Since the small $u$ should contribute to $V_{N}(r)$ dominantly, one can approximately change Eq.(3.17) into

$$
V_{N}(r) \sim \frac{-2}{\pi^{2} M^{3} R r} \int_{0}^{\infty} d u \sin \left(\frac{r}{R} u\right) \frac{u}{3+u^{2}}
$$

which results in

$$
V_{N}(r) \sim-\frac{1}{\pi M^{3} R r} e^{-\sqrt{3} \frac{r}{R}}
$$

at the long-range, i.e. $r>>$. Thus one can conclude that the gravitational force at the Neumann brane is exponentially suppressed. It is in fact conjectured from the fact that $\hat{G}_{1}^{N}[R, R ; E]$ has a pole at $m_{N} \sim 2.48 R^{-1}$, which makes the graviton on the Neumann brane to be massive.

At the short-range one can use the asymptotic formula for the modified Bessel function, which results in

$$
V_{N}(r) \sim \frac{1}{\pi^{2} M^{3} R r}\left[\lim _{\epsilon \rightarrow 0} \int_{0}^{\infty} d u \sin \left(\frac{r}{R} u\right) e^{-\epsilon u}-\frac{3}{2} \int_{0}^{\infty} d u \frac{\sin \left(\frac{r}{R} u\right)}{\frac{3}{2}-u}\right]
$$

where the infinitesimal parameter $\epsilon$ is introduced again for the regularization. Carrying out the integration using the integral formula

$$
\int_{0}^{\infty} \frac{\sin (a x)}{\beta-x} d x=\sin (\beta a) c i(\beta a)-\cos (\beta a)[\operatorname{si}(\beta a)+\pi]
$$

where $\operatorname{si}(z)$ and $c i(z)$ are usual sine and cosine integral respectively, one can express $V_{N}(r)$ at short-range as

$$
V_{N}(r) \sim \frac{1}{\pi^{2} M^{3} R r}\left[\frac{R}{r}-\frac{3}{2}\left\{\sin \left(\frac{3 r}{2 R}\right) c i\left(\frac{3 r}{2 R}\right)-\cos \left(\frac{3 r}{2 R}\right) \operatorname{si}\left(\frac{3 r}{2 R}\right)-\pi \cos \left(\frac{3 r}{2 R}\right)\right\}\right] .
$$

Since $r<<R$, one can expand $V_{N}(r)$ using the following expansions of $\operatorname{si}(z)$ and $\operatorname{ci}(z)[41]$ :

$$
\begin{aligned}
& \operatorname{si}(z)=-\frac{\pi}{2}+\sum_{k=1}^{\infty} \frac{(-1)^{k+1} z^{2 k-1}}{(2 k-1)(2 k-1) !} \\
& \operatorname{ci}(z)=\gamma+\ln z+\sum_{k=1}^{\infty}(-1)^{k} \frac{z^{2 k}}{2 k(2 k) !}
\end{aligned}
$$


where $\gamma=0.577 \cdots$ is an Euler's constant. Then finally $V_{N}(r)$ reduces to

$$
V_{N}(r) \sim \frac{G_{5}}{r^{2}}\left[1-\left(\frac{3 r}{2 R}\right)^{2} \ln \left(\frac{3 r}{2 R}\right)\right]
$$

at short-range, where $5 d$ Newton constant $G_{5}$ is defined as $G_{5}=1 / \pi^{2} M^{3}$. Thus, for the Neumann brane $5 d$ Newton potential arises at the short-range like RS brane. The different point, however, is that $V_{N}(r)$ has a logarithmic sub-leading correction.

Now, finally let us consider $\xi=0$ case which means we have chosen the Dirichlet BC at the brane for the fixed-energy amplitude of $\hat{H}_{0}$ in Eq.(3.1). If one naively inserts $\xi=$ 0 in Eq.(3.2), $\hat{G}_{1}^{D}[a, b ; E]$ becomes to be identical to $\hat{G}_{0}^{D}[a, b ; E]$ because the modification term in Eq.(3.2) arising due to the $\delta$-function potential via Scuulman procedure [39,40] vanishes at $\xi=0$. Even in this case, however, one can generate the non-trivial fixed-energy amplitude through a coupling constant renormalization. In order to adopt this procedure we should regard the coupling constant $v$ as an unphysical and infinite bare one. Then one can introduce a physical renormalized coupling constant $v^{r e n}$ which is related to $v$ by $v^{r e n}=(1 / v-2 \epsilon) / 2 \epsilon^{2}$, where $\epsilon$ is an infinitesimal parameter. Through this renormalization procedure one can derive a non-trivial fixed-energy amplitude $[24,25]^{3}$ :

$$
\hat{G}_{1}^{D, r e n}\left[a, b ; \frac{m^{2}}{2}\right]=\hat{G}_{0}^{D}[a, b ; E]+\frac{2 \sqrt{a b}}{\left(R v^{r e n}+\frac{3}{2}\right)+m R \frac{K_{1}(m R)}{K_{2}(m R)}} \frac{K_{2}(m a) K_{2}(m b)}{K_{2}^{2}(m R)}
$$

which results in

$$
\hat{G}_{1}^{D, r e n}\left[R, R ; \frac{m^{2}}{2}\right]=\frac{2 R}{\left(R v^{r e n}+\frac{3}{2}\right)+m R \frac{K_{1}(m R)}{K_{2}(m R)}} .
$$

Inserting Eq.(3.26) into Eq.(2.12) the Newton potential arising due to the exchange of the gravity localized on the Dirichlet brane becomes

$$
V_{D}(r)=\frac{1}{\pi^{2} M^{3} R r} \int_{0}^{\infty} d u u \sin \left(\frac{r}{R} u\right) \frac{1}{\left(R v^{r e n}+\frac{3}{2}\right)+u \frac{K_{1}(u)}{K_{2}(u)}}
$$

\footnotetext{
${ }^{3}$ there is a factor 2 mistake in Eq.(26) of Ref. [24]
} 
Firstly, let us consider a case of $R v^{r e n}+3 / 2=0$. Comparing Eq.(3.26) in this case with Eq.(3.4) the fixed-energy amplitude $\hat{G}_{1}^{D, r e n}[R, R ; E]$ becomes $2 \hat{G}_{1}^{R S}[R, R ; E]$, which implies $V_{D}(r)=2 V_{R S}(r)$ which is summarized in Eq.(3.14).

Next, let us consider a case of $R v^{r e n}+3 / 2 \neq 0$. Since the small $u$ region contributes dominantly in the long-range, Eq.(3.27) becomes

$$
V_{D}(r) \sim \frac{2}{\pi^{2} M^{3} R r} \int_{0}^{\infty} d u \frac{u \sin \left(\frac{r}{R} u\right)}{u^{2}+\left(2 R v^{r e n}+3\right)}
$$

which reduces to

$$
V_{D}(r) \sim \frac{1}{\pi M^{3} R r} e^{-\sqrt{2 R v^{r e n}+3} \frac{r}{R}}
$$

The expontial suppression of $V_{D}(r)$ in this long-range behavior indicates that the gravity localized on the Dirichlet brane is massive.

If $r<<R$, the same calculation makes $V_{D}(r)$ to be

$$
V_{D}(r) \sim \frac{1}{\pi^{2} M^{3} R r}\left[\lim _{\epsilon \rightarrow 0} \int_{0}^{\infty} d u \sin \left(\frac{r}{R} u\right) e^{-\epsilon u}-\left(R v^{r e n}+\frac{3}{2}\right) \int_{0}^{\infty} d u \frac{\sin \left(\frac{r}{R} u\right)}{u+\left(R v^{r e n}+\frac{3}{2}\right)}\right]
$$

where the infinitesimal parameter $\epsilon$ is introduced again for the regularization. Using the integral formula

$$
\int_{0}^{\infty} \frac{\sin a x}{x+\beta} d x=f(a \beta)
$$

where $f(z) \equiv \operatorname{ci}(z) \sin (z)-\operatorname{si}(z) \cos (z), V_{D}(r)$ becomes

$$
V_{D}(r) \sim \frac{1}{\pi^{2} M^{3} R r}\left[\frac{R}{r}-\left(R v^{r e n}+\frac{3}{2}\right) f\left(\frac{r}{R}\left[R v^{r e n}+\frac{3}{2}\right]\right)\right] .
$$

Thus the short-range behavior of $V_{D}(r)$ is governed by the remormalized coupling constant. If $\frac{r}{R}\left(R v^{r e n}+\frac{3}{2}\right)>>1, V_{D}(r)$ becomes

$$
V_{D}(r) \sim \frac{2 R^{2}\left(R v^{r e n}+\frac{3}{2}\right)^{-2}}{\pi^{2} M^{3} r^{4}}\left[1-\left(R v^{r e n}+\frac{3}{2}\right)^{-2} \frac{12 R^{2}}{r^{2}}\right] .
$$

When deriving Eq.(3.33) we used the asymptotic expression [41]: 


$$
f(z)=\frac{1}{z}\left(1-\frac{2 !}{z^{2}}+\frac{4 !}{z^{2}}-\cdots\right) .
$$

It is interesting to note that the leading order in this case is $1 / r^{4}$ which should be a leading term of the seven-dimensional Newton law. It is unclear at least for us why this pecular behavior of Newton potential arises on Dirichlet brane.

If $\frac{r}{R}\left(R v^{r e n}+\frac{3}{2}\right)<<1$, we should use Eq.(3.23) which results in

$$
V_{D}(r) \sim \frac{1}{\pi^{2} M^{3} r^{2}}\left[1-\frac{\pi}{2}\left(R v^{r e n}+\frac{3}{2}\right) \frac{r}{R}\right]
$$

Thus in this case $V_{D}(r)$ exhibits the $5 d$-type Newton potential.

\section{NEWTON POTENTIAL FROM FIXED-ENERGY AMPLITUDE : WITH $4 D$ INDUCED EINSTEIN TERM}

In this section we will compute the Newton potential on the brane when the $4 d$ induced Einstein term is involved. The remarkable feature in this case is an appearance of the $\delta$-function potential which has an energy(or mass)-dependent coupling constant in the linearized fluctuation equation $[31,35]$ :

$$
\begin{aligned}
& \hat{H}_{2} \hat{\psi}(z)=E \hat{\psi}(z) \\
& \hat{H}_{2}=\hat{H}_{1}+\lambda E \delta(z)
\end{aligned}
$$

where $\lambda \equiv M_{4}^{2} / M^{3}$ and $\hat{H}_{1}$ is defined in Eq.(3.1). Thus, the fixed-energy amplitude for $\hat{H}_{2}$ can be obtained by performing the Schulman procedure $[39,40]$ again

$$
\hat{G}_{2}[a, b ; E]=\hat{G}_{1}[a, b ; E]-\frac{\hat{G}_{1}[a, c ; E] \hat{G}_{1}[c, b ; E]}{\frac{1}{\lambda E}+\hat{G}_{1}[c, c ; E]} .
$$

Inserting $a=b=c$ in Eq.(4.2) yields the fixed-energy amplitude on the brane

$$
\hat{G}_{2}[c, c ; E]=\frac{\frac{1}{\lambda E} \hat{G}_{1}[c, c ; E]}{\frac{1}{\lambda E}+\hat{G}_{1}[c, c ; E]} .
$$

Thus, combining Eq.(2.12) and (4.3) we can compute the Newton potential generated by the exchange of the gravity localized on the brane. 
Firstly, let us consider $\xi=1 / 2$ case in which the fixed-energy amplitude reduces by Eq.(4.3) to

$$
\hat{G}_{2}^{R S}\left[R, R ; \frac{m^{2}}{2}\right]=\frac{2}{m} \frac{K_{2}(m R)}{2 K_{1}(m R)+\lambda m K_{2}(m R)} .
$$

Of course, we used $c=R, g=15 / 8$ and $v=3 / 2 R$ when deriving Eq.(4.4). Inserting Eq.(4.4) into (2.12) the Newton potential becomes

$$
V_{2, R S}(r)=\frac{1}{\pi^{2} M^{3} R r} \int_{0}^{\infty} d u \sin \left(\frac{r}{R} u\right) \frac{K_{2}(u)}{2 K_{1}(u)+\frac{\lambda}{R} u K_{2}(u)} .
$$

Firstly, let us consider the long-range behavior of $V_{2, R S}(r)$. Since small $u$ region contributes dominantly at long-range, one can approximate

$$
\begin{aligned}
& K_{1}(u) \sim \frac{1}{u}+\frac{u}{2} \ln u \\
& K_{2}(u) \sim \frac{1}{u^{2}}-\frac{1}{2}
\end{aligned}
$$

which makes $V_{2, R S}(r)$ to be

$$
\begin{aligned}
V_{2, R S}(r) \sim \frac{1}{\pi^{2} M^{3} R\left(2+\frac{\lambda}{R}\right) r} & {\left[\int_{0}^{\infty} d u \frac{\sin \left(\frac{r}{R} u\right)}{u}\right.} \\
& \left.-\frac{1}{2+\frac{\lambda}{R}} \lim _{\epsilon \rightarrow 0} \int_{0}^{\infty} d u u e^{-\epsilon u} \sin \left(\frac{r}{R} u\right) \ln u\right] .
\end{aligned}
$$

In Eq.(4.7) we introduced again the infinitesimal parameter $\epsilon$ for the proper regularizarion. Making use of Eq.(3.11), one can show that $V_{2, R S}(r)$ at long-range obeys a $4 d$ Newton law with $1 / r^{3}$ subleading correction as following:

$$
V_{2, R S}(r) \sim \frac{1}{2 \pi M^{3} R\left(2+\frac{\lambda}{R}\right) r}\left[1+\frac{R^{2}}{\left(2+\frac{\lambda}{R}\right) r^{2}}\right] .
$$

Next, let us examine the short-range behavior of $V_{2, R S}(r)$. Since the large $u$ region makes a dominant contribution to $V_{2, R S}(r)$, the asymptotic formulae

$$
\begin{aligned}
& K_{1}(u) \sim \sqrt{\frac{\pi}{2 u}} e^{-u}\left(1+\frac{3}{8 u}\right) \\
& K_{2}(u) \sim \sqrt{\frac{\pi}{2 u}} e^{-u}\left(1+\frac{15}{8 u}\right)
\end{aligned}
$$


can be used. Then one can change $V_{2, R S}(r)$ into

$$
\begin{aligned}
V_{2, R S}(r) & =\frac{1}{\pi^{2} M^{3} \lambda r}\left[\int_{0}^{\infty} \frac{\sin \left(\frac{r}{R} u\right)}{u+\frac{R}{\lambda}\left(2+\frac{15 \lambda}{8 R}\right)} d u\right. \\
& \left.+\frac{15}{8} \frac{1}{\frac{R}{\lambda}\left(2+\frac{15 \lambda}{8 R}\right)}\left\{\int_{0}^{\infty} \frac{\sin \left(\frac{r}{R} u\right)}{u} d u-\int_{0}^{\infty} \frac{\sin \left(\frac{r}{R} u\right)}{u+\frac{R}{\lambda}\left(2+\frac{15 \lambda}{8 R}\right)} d u\right\}\right] .
\end{aligned}
$$

Making use of the integral formula (3.31), $V_{2, R S}(r)$ at $r<<R$ reduces to

$$
V_{2, R S}(r) \sim \frac{1}{\pi^{2} M^{3} \lambda r}\left[f\left(\frac{2 r}{\lambda}+\frac{15 r}{8 R}\right)+\frac{15}{8} \frac{1}{\frac{R}{\lambda}\left(2+\frac{15 \lambda}{8 R}\right)}\left\{\frac{\pi}{2}-f\left(\frac{2 r}{\lambda}+\frac{15 r}{8 R}\right)\right\}\right] .
$$

If the quantum effect of the one loop is so large, i.e. $\lambda>>R>>r$, the Newton potential $V_{2, R S}(r)$ becomes

$$
V_{2, R S}(r) \sim \frac{1}{\pi^{2} M^{3} \lambda r}\left[f\left(\frac{15 r}{8 R}\right)+\frac{15}{8} \frac{1}{\frac{15}{8}+\frac{2 R}{\lambda}}\left\{\frac{\pi}{2}-f\left(\frac{15 r}{8 R}\right)\right\}\right],
$$

which reduces to approximately

$$
V_{2, R S}(r) \sim \frac{1}{2 \pi M^{3} \lambda r}\left[1+\frac{4 r}{\pi \lambda} \ln \left(\frac{15 r}{8 R}\right)\right]
$$

Thus, the Newton potential recovers the $4 d$-type $1 / r$ gravitational potential. If the quantum effect of the one loop is very small compared to $R$, i.e. $\lambda<<R$, Eq.(4.11) becomes

$$
V_{2, R S}(r) \sim \frac{1}{\pi^{2} M^{3} \lambda r} f\left(\frac{2 r}{\lambda}\right)
$$

Thus in the region $\lambda<<r<<R$, which means the quantum effect is extremely small, the Newton potential exhibits a $5 d$-type potential with $1 / r^{4}$ correction term as following

$$
V_{2, R S}(r) \sim \frac{1}{2 \pi^{2} M^{3} r^{2}}\left(1-\frac{\lambda^{2}}{2 r^{2}}\right)
$$

When deriving Eq.(4.15) we have used the expansion (3.34). If $r<<\lambda<<R$, we should use the expansion (3.23), which results in

$$
V_{2, R S}(r) \sim \frac{1}{2 \pi M^{3} \lambda r}\left(1+\frac{4 r}{\pi \lambda} \ln \frac{2 r}{\lambda}\right) .
$$

Thus at the extremely small-range the $4 d$ Newton law is recovered. This result is also confirmed in Ref. [31,35]. 
In appendix $\mathrm{A}$ we examined the Newton potential when $\xi=1$. Let us summarize the result. The long-range behavior in this case is an exponential suppression like the picture without the $4 d$ induced Einstein term:

$$
V_{2, N}(r) \sim-\frac{1}{\pi M^{3}(R+2 \lambda) r} \exp \left\{-\frac{r}{R} \sqrt{\frac{3}{1+\frac{2 \lambda}{R}}}\right\} .
$$

This indicates that the graviton localized on the Neumann brane is massive. In the region of $\lambda<<r<<R$ the Newton potential shows the $5 d$-type with $1 / r^{4}$ subleading correction:

$$
V_{2, N}(r) \sim \frac{1}{\pi^{2} M^{3} r^{2}}\left(1-\frac{2 \lambda^{2}}{r^{2}}\right) .
$$

However, at the extremely short-range, i.e. $r<<\lambda<<R$, the potential recovers the $4 d$-type Newton law:

$$
V_{2, N}(r) \sim \frac{1}{2 \pi M^{3} \lambda r}\left(1+\frac{2 r}{\pi \lambda} \ln \frac{r}{\lambda}\right)
$$

In appendix $\mathrm{B}$ we compute the Newton potential at $\xi=0$. In this case the fixed-energy amplitude as well as the Newton potential depend on the renormalized coupling constant $v^{r e n}$ as in the case without the $4 d$ induced Einstein term. If $R v^{r e n}+\frac{3}{2}=0$, the final expression of the Newton potential is related to $V_{2, R S}(r)$ as following:

$$
V_{2, D}(r)=\left.2 V_{2, R S}(r)\right|_{\lambda \rightarrow 2 \lambda}
$$

If $R v^{r e n}+\frac{3}{2} \neq 0$, Newton potential is exponentially suppressed in the long-range as the case in the absence of the $4 d$ induced Einstein term as following:

$$
V_{2, D}(r) \sim \frac{1}{2 \pi M^{3}\left(\lambda+\frac{R}{2}\right) r} \exp \left\{-\frac{r}{R} \sqrt{\frac{R v^{r e n}+\frac{3}{2}}{\frac{1}{2}+\frac{\lambda}{R}}}\right\} .
$$

This indicates that the graviton localized on the Dirichlet brane is massive. In the shortrange the Newton potential becomes

$$
V_{2, D}(r) \sim \frac{1}{\pi^{2} M^{3} r^{2}}\left(1-\frac{2 \lambda^{2}}{r^{2}}\right)
$$

when $\lambda<<r<R$ and 


$$
V_{2, D}(r) \sim \frac{1}{2 \pi M^{3} \lambda r}\left(1+\frac{2 r}{\pi \lambda} \ln \frac{r}{\lambda}\right)
$$

when $r<<\lambda<<$. Thus, we have an intermediate range $\lambda<<r<<R$ in which the $5 d$ Newton law emerges. It is interesting to note that $V_{2, D}(r)$ at short-range is independent of the renormalized coupling constant $v^{r e n}$. Furthermore, the potential on Dirichlet brane coincides with that on Neumann brane in the short-range.

\section{CONCLUSION}

In this paper we examined Newton law generated by the localized gravity on the general singular brane when the bulk spacetime is a single copy of $A d S_{5}$. We used a fixed-energy amplitudes which are obtained from the linearized gravitational fluctuation equation by applying the technique of SQM. Since the bulk is a single copy of $A d S_{5}$, SQM naturally makes the fixed-energy amplitude to be dependent on the $\mathrm{BC}$ at the location of the brane. We examined Newton potential on $\operatorname{RS} \operatorname{brane}(\xi=1 / 2)$, Neumann brane $(\xi=1)$, and Dirichlet $\operatorname{brane}(\xi=0)$.

For RS brane the usual RS scenario gives rise to the $4 d$-type Newton potential at longrange and 5d-type at short-range. However, the $4 d$ Einstein term induced by a quantum effect of one-loop changes the general behavior of Newton potential. In this case there is an intermediate range $\lambda<<r<<$, in which Newton potential is five-dimensional. At other ranges the four-dimensional Newton law is recovered.

For Neumann brane the long-range Newton potential is exponentially suppressed regardless of the existence of the $4 d$ induced Einstein term. This is because the gravity localized on the Neumann brane acquires a mass. In the short-range Newton potential becomes five-dimensional with a logarithmic subleading correction if there is no $4 d$ induced Einstein term. The Einstein term in Neumann brane yields a similar intermediate range to that in RS brane, where Newton law is five-diemnsional.

For Dirichlet brane the non-trivial fixed-energy amplitude can be derived via the coupling constant renormalization scheme. Thus, Newton potential on the Dirichlet brane is usually 
dependent on the renormalized coupling constant $v^{r e n}$. If $v^{r e n}=-3 / 2 R$, the final expression of Newton potential is proportional to that in $\mathrm{RS}$ brane. If $v^{\text {ren }} \neq-3 / 2 R$, the long-range behavior of Newton potential is expontially suppressed due to the massive gravity regardless of the $4 d$ induced Einstein term. In short-range Newton potential exhibits an pecular $1 / r^{4}$ behavior if $\frac{r}{R}\left(R v^{r e n}+\frac{3}{2}\right)>>1$. However, the $4 d$ Einstein term makes the short-range behavior of Newton potential on Dirichlet brane to be exactly identical to that on Neumann brane.

Recently, Newton law arising due to the localized gravity on various curved brane is examined when bulk is $d S_{5}$ or $A d S_{5}$ [42]. Our SQM technique may be applied straightforwardly to these various scenarios. The explicit result will be given elsewhere.

Acknowledgement: This work was supported by the Kyungnam University Research Fund, 2002. 


\section{REFERENCES}

[1] H. C. Lee (Ed.), An introduction to Kaluza-Klein Theories (World Scientific, Singapore, 1984).

[2] V. A. Rubakov and M.E. Shaposhnikov, Phys. Lett. B125 (1983) 139.

[3] M. Visser, Phys. Lett. B159 (1985) 22.

[4] N. Arkani-Hamed, S. Dimopoulos and G. Dvali, Phys. Lett. B429 (1998) 263 [hepph/9803315].

[5] L. Antoniadis, N. Arkani-Hamed, S. Dimopoulos and G. Dvali, Phys. Lett. B436 (1998) 257 [hep-ph/9804398].

[6] L. Randall and R. Sundrum, Phys. Rev. Lett. 83 (1999) 3370 [hep-ph/9905221].

[7] L. Randall and R. Sundrum, Phys. Rev. Lett. 83 (1999) 4690 [hep-th/9906064].

[8] P. Horava and E. Witten, Nucl. Phys. B460 (1996) 506 [hep-th/9510209].

[9] P. Binetruy, C. Deffayet and D. Langlois, Nucl. Phys. B565 (2000) 269 [hepth/9905012].

[10] C. Csáki, M. Graesser, C. Kolda and J. Terning, Phys. Lett. B462 (1999) 34 [hepph/9906513].

[11] J. M. Cline, C. Grojean and G. Servant, Phys. Rev. Lett. 83 (1999) 4245 [hep$\mathrm{ph} / 9906523]$.

[12] J. E. Kim, B. Kyae and H. M. Lee, Phys. Rev. Lett. 86, (2001) 4223 [hep-th/0011118].

[13] S. Alexander, Y. Ling and L. Smolin, Phys. Rev. D65 (2002) 083503 [hep-th/0106097].

[14] D. K. Park, H. S. Kim and S. Tamaryan, Phys. Lett. B535 (2002) 5 [hep-th/0111081].

[15] N. Arkani-Hamed, H. C. Cheng, P. Creminelli and L. Randall, hep-th/0302034 and references therein. 
[16] A. Chamblin, S. W. Hawking and H. S. Reall, Phys. Rev. D61 (2000) 065007 [hepth/9909205].

[17] R. Emparan, G. T. Horowitz and R. C. Myers, JHEP 0001 (2000) 007 [hep-th/9911043].

[18] S. B. Giddings, E. Katz and L. Randall, JHEP 0003 (2000) 023 [hep-th/0002091].

[19] S. B. Giddings and S. Thomas, Phys. Rev. D65 (2002) 056010 [hep-th/0106219].

[20] S. B. Giddings, hep-th/0205027.

[21] S. B. Giddings, Gen. Rel. Grav. 34 (2002) 1775 [hep-th/0205205].

[22] J. Garriga and T. Tanaka, Phys. Rev. Lett. 84 (2000) 2778 [hep-th/9911055].

[23] M. J. Duff and J. T. Liu, Phys. Rev. Lett. 85 (2000) 2052 [hep-th/0003237].

[24] D. K. Park and S. Tamaryan, Phys. Lett. B532 (2002) 305 [hep-th/0108068].

[25] D. K. Park and H. S. Kim, Nucl. Phys. B 636 (2002) 179 [hep-th/0204122].

[26] A. Z. Capri, Nonrelativistic Quantum Mechanics (Benjamin/Cummings, Menlo Park, CA, 1985).

[27] M. Reed and B. Simon, Methods of Modern Mathematical Physics (Academic, New York, 1975).

[28] R. Jackiw, in M. A. Bég Memorial Volume, edited by A. Ali and P. Hoodbhoy (World Scientific, Singapore, 1991).

[29] D. K. Park, J. Math. Phys. 36 (1995) 5453 [hep-th/9405020].

[30] D. K. Park and S. Tamaryan, Phys. Lett. B 554 (2003) 92 [hep-th/0212023].

[31] D. K. Park, Phys. Lett. B 562 (2003) 316 [hep-th/0304056].

[32] G. Dvali, G. Gabadadze and M. Porrati, Phys. Lett. B 485 (2000) 208 [hep-th/0005016].

[33] G. Dvali, G. Gabadadze, M. Kolanović and F. Nitti, Phys. Rev. D 64 (2001) 084004 
[hep-ph/0102216].

[34] V. A. Rubakov, hep-th/0303125.

[35] E. Kiritsis, N. Tetradis and T. N. Tomaras, JHEP 0203 (2002) 019 [hep-th/0202037].

[36] M. Ito, Phys. Lett. B 554 (2003) 180 [hep-th/0211268].

[37] J. Maldacena, Adv. Theor. Math. Phys. 2 (1998) 231 [hep-th/9711200].

[38] O. Aharony, S. S. Gubser, J. Maldacena, H. Ooguri and Y. Oz, Phys. Rep. 323 (2000) 183 [hep-th/9905111].

[39] B. Gaveau and L. S. Schulman, J. Phys. A 19 (1986) 1833.

[40] L. S. Schulman, in Path Integral from mev to MeV, edited by M. C. Gutzwiller, A. Inomata, J. R. Klauder and L. Streit (World Scientific, Singapore, 1986).

[41] M. Abramowitz and I. A. Stegun, Handbook of Mathematical Functions (Dover, New York, NY, 1972).

[42] S. Nojiri and S. D. Odintsov, Phys. Lett. B 548 (2002) 215 [hep-th/0209066] and references therein. 


\section{Appendix A}

In this appendix we will examine the Newton potential arising due to the gravity localized on the Neumann brane when the $4 d$ induced Einstein term exists. The fixed-energy amplitude for $\xi=1$ with $c=R, g=15 / 8$ and $v=3 / 2 R$ yields

$$
\hat{G}_{2}^{N}\left[R, R ; \frac{m^{2}}{2}\right]=\frac{\frac{2}{m} \frac{K_{2}(m R)}{K_{1}(m R)}}{1+\left(\lambda m-\frac{3}{2 m R}\right) \frac{K_{2}(m R)}{K_{1}(m R)}}
$$

and the corresponding Newton potential derived from Eq.(2.12) is

$$
V_{2, N}(r)=\frac{1}{\pi^{2} M^{3} R r} \int_{0}^{\infty} d u \sin \left(\frac{r}{R} u\right) \frac{\frac{K_{2}(u)}{K_{1}(u)}}{1+\left(\frac{\lambda}{R} u-\frac{3}{2 u}\right) \frac{K_{2}(u)}{K_{1}(u)}} .
$$

If two unit masses are separated at long distance compared to the $A d S_{5}$ radius, i.e. $r>>R$, the small $u$ region contributes to $V_{2, N}(r)$ dominantly. Thus we can use an expansion (4.6) for the modified Bessel function, which results in approximately

$$
V_{2, N}(r) \sim-\frac{2}{\pi^{2} M^{3}(R+2 \lambda) r} \int_{0}^{\infty} d u \sin \left(\frac{r}{R} u\right) \frac{u}{\frac{3}{1+\frac{2 \lambda}{R}}+u^{2}} .
$$

Making use of the integral formula

$$
\int_{0}^{\infty} \frac{x \sin (a x)}{\beta^{2}+x^{2}} d x=\frac{\pi}{2} e^{-a \beta}
$$

one can show that the gravitational force on the Neumann brane is exponentially suppressed as following:

$$
V_{2, N}(r) \sim-\frac{1}{\pi M^{3}(R+2 \lambda) r} \exp \left\{-\frac{r}{R} \sqrt{\frac{3}{1+\frac{2 \lambda}{R}}}\right\} .
$$

If two unit masses are separated at short distance, the large $u$ region contributes domi-

nantly. Thus we can use the asymptotic formula (4.9), which results in the following Newton potential

$$
\begin{aligned}
V_{2, N}(r) \sim \frac{1}{\pi^{2} M^{3} \lambda r}\left[\int_{0}^{\infty} \frac{\sin \left(\frac{r}{R} u\right)}{u+\left(\frac{15}{8}+\frac{R}{\lambda}\right)}\right. \\
\left.+\frac{\frac{15}{8}}{\frac{15}{8}+\frac{R}{\lambda}}\left\{\int_{0}^{\infty} d u \frac{\sin \left(\frac{r}{R} u\right)}{u}-\int_{0}^{\infty} d u \frac{\sin \left(\frac{r}{R} u\right)}{u+\left(\frac{15}{8}+\frac{R}{\lambda}\right)}\right\}\right] .
\end{aligned}
$$


Computing the integrations of Eq.(A.6) with use of Eq.(3.31), one can express $V_{2, N}(r)$ at short-range as

$$
V_{2, N}(r) \sim \frac{1}{\pi^{2} M^{3} \lambda r}\left[f\left(\frac{15 r}{8 R}+\frac{r}{\lambda}\right)+\frac{\frac{15}{8}}{\frac{15}{8}+\frac{R}{\lambda}}\left\{\frac{\pi}{2}-f\left(\frac{15 r}{8 R}+\frac{r}{\lambda}\right)\right\}\right] .
$$

If $\lambda<<R, V_{2, N}(r)$ in Eq.(A.7) becomes

$$
V_{2, N}(r) \sim \frac{1}{\pi^{2} M^{3} \lambda r} f\left(\frac{15 r}{8 R}+\frac{r}{\lambda}\right)
$$

Thus using the expansion of the sine and cosine integral functions (3.23) and (3.34), the Newton potential on the Neumann brane becomes

$$
V_{2, N}(r) \sim \frac{1}{\pi^{2} M^{3} r^{2}}\left(1-\frac{2 \lambda^{2}}{r^{2}}\right)
$$

in the region of $\lambda<<r<<R$, and

$$
V_{2, N}(r) \sim \frac{1}{2 \pi M^{3} \lambda r}\left(1+\frac{2 r}{\pi \lambda} \ln \frac{r}{\lambda}\right)
$$

in the region of $r<<\lambda<<R$.

\section{Appendix B}

In this appendix we will examine the Newton potential arising due to the gravity localized on the Dirichlet brane when the $4 d$ induced Einstein term exists. The fixed-energy amplitude

for $\xi=0$ case can be obtained from Eq.(4.3) with replacing $\hat{G}_{1}[c, c ; E]$ by a renormalized fixed-energy amplitude $\hat{G}_{1}^{r e n}[c, c ; E]$. Then using Eq.(3.26) one can easily calculate the fixedenergy amplitude

$$
\hat{G}_{2}^{D}\left[R, R ; \frac{m^{2}}{2}\right]=\frac{2 R}{\left(R v^{r e n}+\frac{3}{2}\right)+m R \frac{K_{1}(m R)}{K_{2}(m R)}+R \lambda m^{2}}
$$

which leads the Newton potential to

$$
V_{2, D}(r)=\frac{1}{\pi^{2} M^{3} R r} \int_{0}^{\infty} d u \frac{u \sin \left(\frac{r}{R} u\right)}{\left(R v^{r e n}+\frac{3}{2}\right)+u \frac{K_{1}(u)}{K_{2}(u)}+\frac{\lambda}{R} u^{2}} .
$$


Firstly, let us examine the case of $R v^{r e n}+\frac{3}{2}=0$. Comparing Eq.(B.1) with Eq.(4.4) one can conclude

$$
\hat{G}_{2}^{D}\left[R, R ; \frac{m^{2}}{2}\right]=\left.2 \hat{G}_{2}^{R S}\left[R, R ; \frac{m^{2}}{2}\right]\right|_{\lambda \rightarrow 2 \lambda}
$$

Thus, the Newton potential $V_{2, D}(r)$ in this case can be read from $V_{2, R S}(r)$ as following:

$$
V_{2, D}(r)=\left.2 V_{2, R S}(r)\right|_{\lambda \rightarrow 2 \lambda}
$$

Next, let us examine the case of $R v^{r e n}+\frac{3}{2} \neq 0$. Using the expansion (4.6) one can show $V_{2, D}(r)$ reduces to in the long-range, i.e. $r>>R$,

$$
V_{2, D}(r) \sim \frac{1}{\pi^{2} M^{3}\left(\lambda+\frac{R}{2}\right) r} \int_{0}^{\infty} d u \frac{u \sin \left(\frac{r}{R} u\right)}{\frac{R v^{r e n}+\frac{3}{2}}{\frac{1}{2}+\frac{\lambda}{R}}+u^{2}},
$$

which exhibits an exponential suppression

$$
V_{2, D}(r) \sim \frac{1}{2 \pi M^{3}\left(\lambda+\frac{R}{2}\right) r} \exp \left\{-\frac{r}{R} \sqrt{\frac{R v^{r e n}+\frac{3}{2}}{\frac{1}{2}+\frac{\lambda}{R}}}\right\} .
$$

In the short-range we should use the expansion (4.9) which makes $V_{2, D}(r)$ to be

$$
\begin{aligned}
V_{2, D}(r) \sim \frac{1}{\pi^{2} M^{3} \lambda r}\left[\int_{0}^{\infty}\right. & d u \frac{\sin \left(\frac{r}{R} u\right)}{u+\left(\frac{15}{8}+\frac{R}{\lambda}\right)} \\
& \left.+\frac{\frac{15}{8}}{\frac{15}{8}+\frac{R}{\lambda}}\left\{\int_{0}^{\infty} d u \frac{\sin \left(\frac{r}{R} u\right)}{u}-\int_{0}^{\infty} d u \frac{\sin \left(\frac{r}{R} u\right)}{u+\left(\frac{15}{8}+\frac{R}{\lambda}\right)}\right\}\right] .
\end{aligned}
$$

It is interesting to note that $V_{2, D}(r)$ is independent of the renormalized coupling constant $v^{r e n}$. Furthermore, $V_{2, D}(r)$ in Eq.(B.7) is exactly same with $V_{2, N}(r)$ in Eq.(A.6). Thus the short-range behavior of $V_{2, D}(r)$ should be same with that of $V_{2, N}(r)$. 


\section{FIGURES}

FIG. 1. Plot of $J$ and $\pi R^{2} / 2 r^{2}$ with choosing $\epsilon=0.003$. This figure indicates $J$ and $\pi R^{2} / 2 r^{2}$ merge with each other at $r \sim 15 R$. Thus the long-range behavior of $J$ in Eq.(3.13) is confirmed.

FIG. 2. Plot of $J$ and $R / r$ with choosing $\epsilon=0.003$. This figure indicates $J$ and $R / r$ merge with each other at $r \sim 0.05 R$. Thus the short-range behavior of $J$ in Eq.(3.13) is confirmed. 


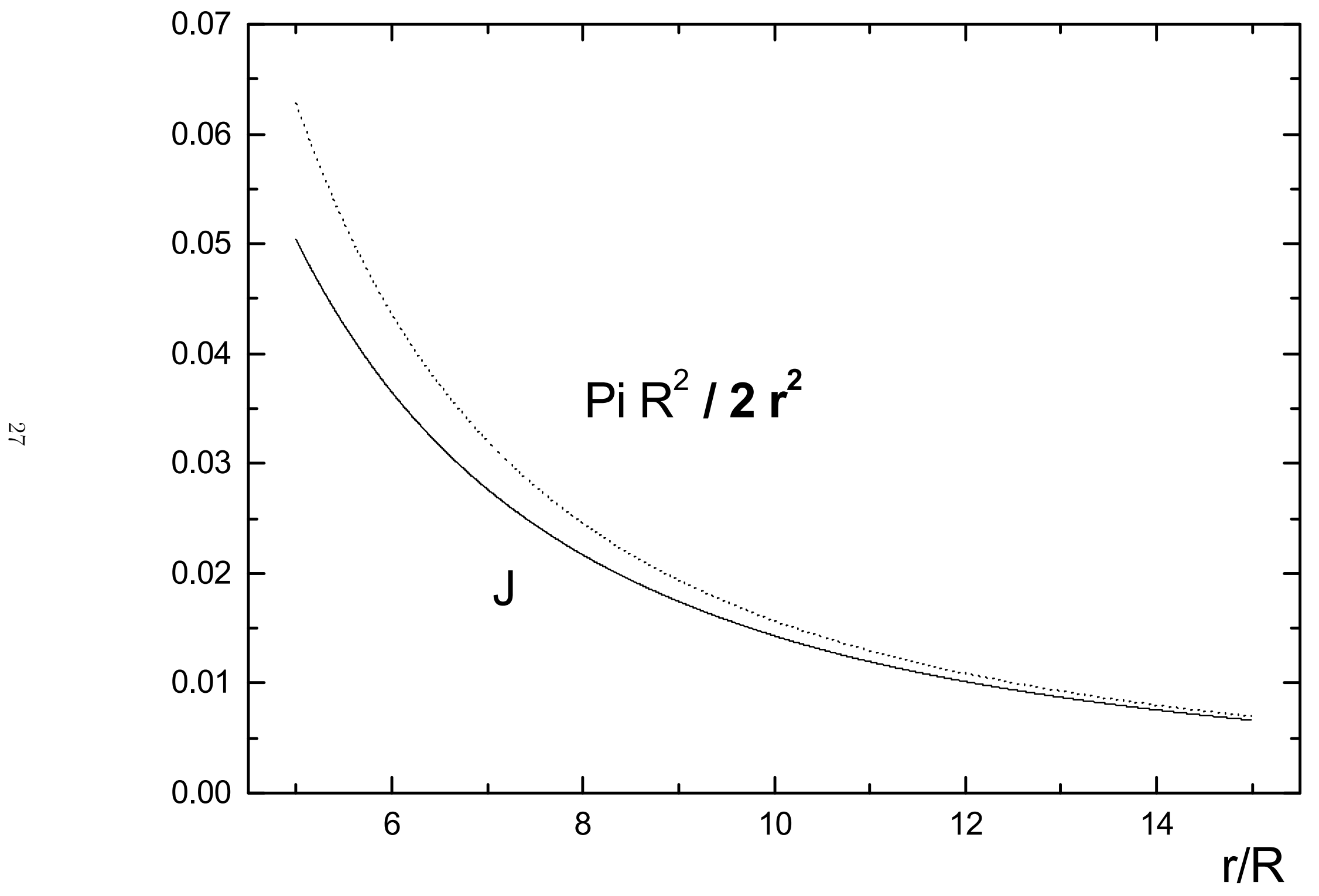

Fig. 1 


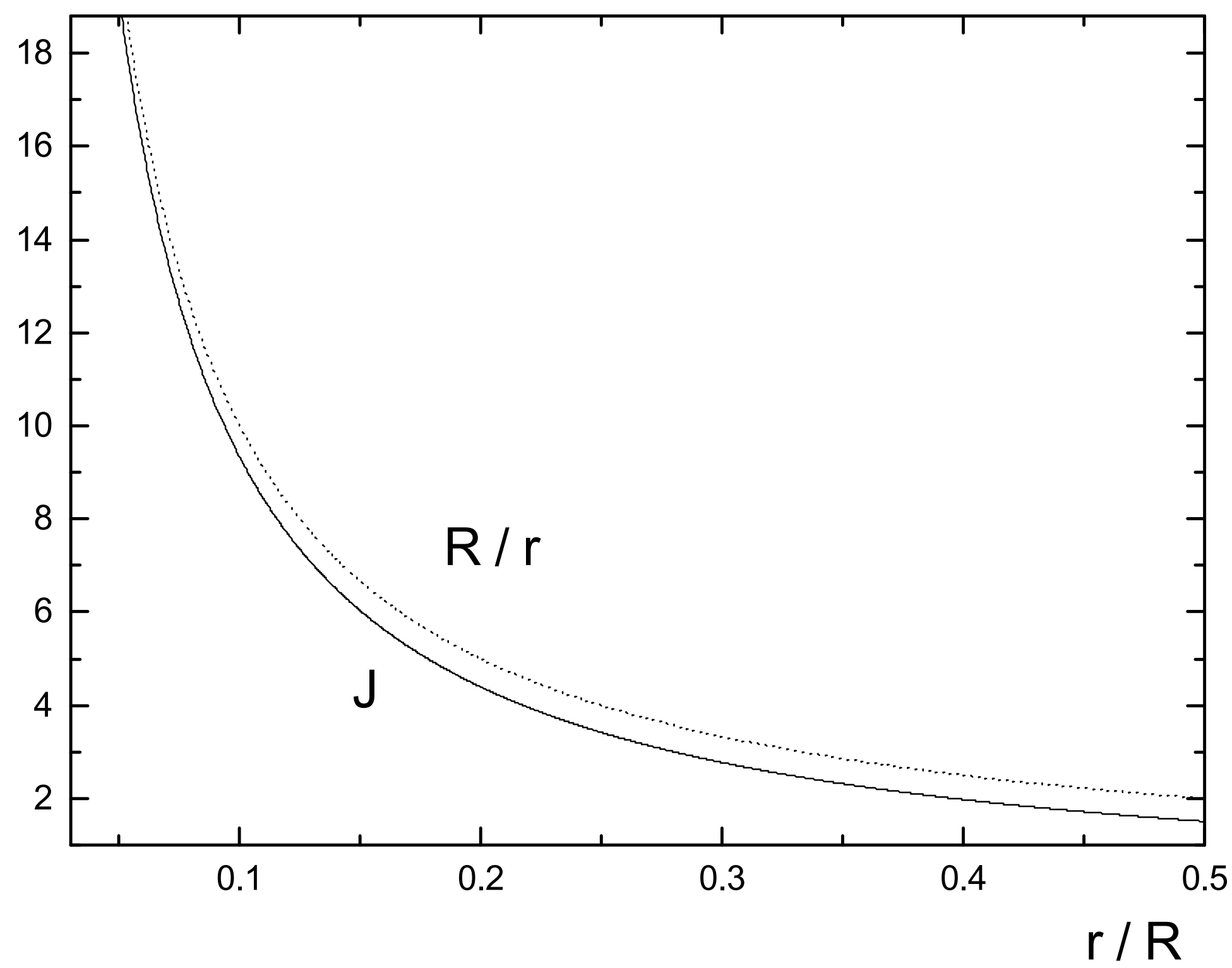

Fig.2 